Vol. 5, No. 2 | July - December 2021

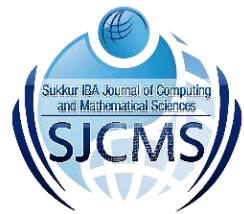

\title{
Orthogonal Zones for Interference Migration in 2.4 GHz Mesh Backhaul
}

\author{
Shazia Abbasi ${ }^{1}$, Muhammad Memon ${ }^{1}$, Khalil Khoumbati ${ }^{2}$, Shahzad Memon ${ }^{1}$
}

\begin{abstract}
:
Managing interference in the multi-radio networks is critical challenge; problem becomes even more serious in $2.4 \mathrm{GHz}$ band due to minimal availability of orthogonal channels. This work attempts to propose a channel assignment scheme for interference zones of $2.4 \mathrm{GHz}$ backhaul of Wireless Mesh Networks (WMN). The static nodes of Infrastructure based Backhaul employing directional antennas to connect static nodes, orthogonal channel zones introducing Interference are formatted with the selection of single tire direct hop and two-tier directional hopes. The effort maintains the orthogonality of channels on system thus reduce the co-channel interference between inter flow and intra flow links. Group of non-overlapping channels of selected band are obtained by a mathematical procedure, interference is modeled by directed graph and Channel assignment is carried out with the help of greedy algorithms. Experimental analysis of the technical proposal is done by simulation through OPNET 14. Our framework can act as an imperative way to enhance the network performance resulting a leading improvement in system throughput and reduction in system delay
\end{abstract}

Keywords: Wireless Mesh Networks (WMN), Backhaul, Orthogonal,Non-orthogonal

\section{Introduction}

The wireless networks are going to restructure for the ultimate communication service comparable in the performance of wired Ethernet [1], [2]. Advance wireless networks support some resource allocation procedures for the effective utilization of spectrum [3], which makes efficient use the simultaneous communication channels of spectrum; interference has been a central issue in this type of networking, There is a possibility that same channel allocate to several nodes which create co-channel interference between simultaneous communications, or there may be adjacent channel interference when adjoining channels allocate to neighboring nodes [4]. In such conditions WMN backhaul is a potential

${ }^{1}$ Faculty of Engineering and Technology Institute of Business studies

${ }^{2}$ University of Sindh, Jamshoro

Corresponding Author: shazia.abbasi@usindh.edu.pk network to offer preferred performance [5], the fact is only to observer

and manages the backhaul links to reduce interference [6]. In the condition of WMN infrastructure, backhaul links are concurrently working on dissimilar channels; the arrangement of channels for backhaul links may enhance or reduce interference of system [7]. However, if the channels are not properly assigned to nodes then the performance of system drops proportionally with the amount of nodes and interfaces. Interference in any form can be reduced by using a suitable arrangement for the allocation of channels.

In general, two methods are used for the allocation of channels: Static channel (SC) assignment and dynamic channel (DC) assignment. Dynamic scheme support the mechanism where channels assign to

$$
\text { SJCMS | P-ISSN: 2520-0755| E-ISSN: 2522-3003 | Vol. } 5 \text { No. } 2 \text { July - December } 2021
$$


interfaces animatedly [8], nodes have ability to sweep channels to meet the required criteria of system, in this respect dynamic channel assignment is effective in use for mobile communication, on the other hand static allocation of channels is suitable for fixed nodes [9], under static allocation, channels allotted to interfaces, remains permanent and fixed for required time. In our previous work, the employed strategy was used by backhaul links of Wireless local area network [10], here the proposed strategy is employed on infrastructure based WMN backhaul. The backhaul is working on $2.4 \mathrm{GHz}$ band and linked by directional antennas. The key consideration of this work is formatting the interference zones and selection of orthogonal channels for such zones to reduce co channel interference, whereas improper (nonorthogonal) arrangement of channels under directional antennas creates serious level of interference as the signals would have more power in particular direction and covers maximum areas. To cover such parameter the zones of interference in proposed to covers both inter-flow links (single tier of direct hop), and intra-flow links (two tiers of directional hop) of directional antenna.

In doing so this paper presents 7 sections. Section 2 presents review of the literature; section 3 provides mathematical procedure to find orthogonal sets. Section 4 discusses about the modelling of the proposed system. Section 5 presents greedy algorithm for channel assignment. Results and discussion on orthogonal channel zones of $2.4 \mathrm{GHz}$ system is presented in section 6. Finally, section 7 provide conclusion.

\section{Literature Review}

The litratutre review reflects lot of research work to deal the problem of interfernce introduced by partially overlapped channels. The concept of utilizing Partially overlapped channels with the alteration of energy masks was presented by the authors of [11], [12], [13]. This technique introduces, loss of information relative to the overlapped frequency range of partially overlapped channels. In other work, orthogonal channels are modeled as Least Congested Channel Search (LCCS), where periodically channel's traffic is observed, in the case of overflow of traffic from threshold level, channel sweeps to LLC [14]. Their work was limited and does not support sudden change of traffic. The work of [15], tried to solve the problem of interfernce in multi channel (POC) environment by multicasting. They employed rational nodes to form multicast trees from soure to destination. Every node of tree, eqqiped with gateway which reduce the interference by limitting the broadcasting of POS channels. Their work aspects the bottelneck problem in heavy traffic. The bottel neck problem is some how overcomed the recent work of [16] where links were ranked on mobility data. The mobility data derived by traffic load and topology of network. Ranking links through multiple data types interduce system latency. The work of [17 and 18] focused on Interference of directional antennas in multi hop networks, Interference among the signals of directional antennas are measured in [17], both antennas are at 90 degree phase change in alignment the work did not contains data regarding spatial separation. The Performance of $802.11 \mathrm{~g}$ system is observed with the support of multi radio [18], they try to prove with quantity study that the overall network efficiency is depended on the employment position of directional antennas, the position consider antennas alignment and distance. Algorithm "directionality as needed (DAN)", is presented in [19], this work meets two aims: network with minimum interference with limited budget. The work of [20] presents the behavior of channel assignment in the environment of directional antennas. They present Outdoor testbed-based measurement of $802.11 \mathrm{a}$, the system connected by directional antenna, in that system they estimate adjacent channel interference with the help of the antenna's physical position, transmitted power of the interfaces and WMN throughput. Research work of [21], [22], [23] assignment of partially overlapped channels discuss about interfering signal and noise ratio (SINR) based novel model for channel allocation, the authors considered that the use of the channel partiality at some affordable 
interference focused collective interference. Therefore, they are able to model only physical interference. A MICA algorithm-based allocation of partially Overlapped Channels (POS) is presented in [22], their focus was to enhance performance while decreasing interference of system. They sustain channel separation and node orthogonality with the consideration of Physical distance of nodes. Another technique to reduce interference is adopted in [23], where alternate channels in the form of odd number are selected for allocation, their results are equivalent with channel assignment where only limited orthogonal channel are used. A greedy channel assignment algorithm for Multi radio Multi Channel WMN is presented in [24] the total interference of static nodes is approximated and assigned channels with the nodes having minimum interference. The suggested work in this paper solved the bottel neck problem by directional antennas at physical layer with minimum latency. Static channels assign to perticular antenna this maintain the orthogonality and channel spacing between channels. Thus there was no loss of energy produced by masking of energy.

\section{Mathematical Procedure to Find Groups of Orthogonal Channels}

Traditionaly the orthogonal channels are drived by using energy masks [11,12,13], discussed in litrature review. This process consumes times and introduce losses of energy w.r.t overlapped portion of two channels. Here a set theory is used to find out the groups of non-overlapped and overlapped channels for the (channel 1 to channel 11) on frequency band $2.4 \mathrm{GHz}$. Universal set " $\mathrm{S}$ ", of $2.4 \mathrm{GHz}$ band is combination of two sets: Orthogonal and Non-orthogonal.

$\mathrm{S}=\{$ Orthogonal channels, Non-orthogonal channels \}

$$
\mathrm{S}=\{\mathrm{On}, \mathrm{NOn}\}
$$

Orthogonal channels "O”, contain all nonoverlapped channels related to the channel number " $n$ " can be find out as,

$$
\mathrm{On}=\mathrm{UN}
$$

$\mathrm{m}=1=\{|(\mathrm{m}-\mathrm{n})|>4\}$

Non-orthogonal channels "NO", contain all overlapped channels related to channel number "n", and can be find out as,

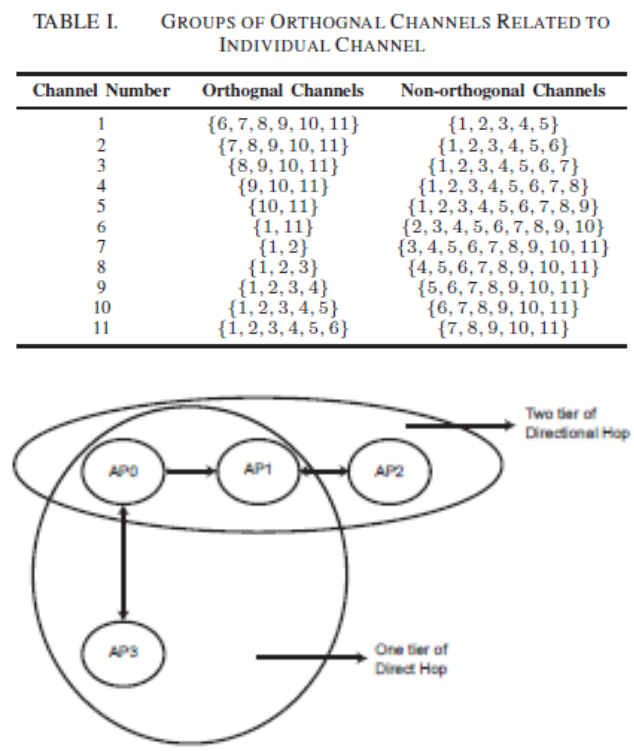

Fig. 1. Interference zone of different AP's NOn $=\{\mathrm{S} \backslash \mathrm{On}\}$

\section{Modelling of Proposed System}

The network graph $\mathrm{G}$ for proposed work is identified as $\mathrm{G}=(\mathrm{U}, \mathrm{V}, \mathrm{L})$. The network consists of $\mathrm{n}$ mesh routers, distributed uniformly and independently over unit area. All nodes are connected by directional antennas for backhaul connectivity. The Area is divided into non overlapping directional

Interference zones "Iz". The system must satisfy the following considerations.

- Number of interference zones = number of interfaces for backhaul connectivity

- Beamwidth of directional zone $=360 \mathrm{deg}$ / number of zones

- Linear area of zones $=2$ directional nodes (2 Tier of directional hop)

- Vertical area of zone $=1$ direct node (single tire of direct hop)

- Minimum number of zones $=1$ 
Area of system is divided in zones, equal to the number of interfaces of node, each zone has a mesh router that is responsible for serving clients within the zone and linked with other zones by directional antenna. To deal with interferences, it is important to identify the interference nodes in Interference zones "Iz" in the network. Work considered two sources of interference, directional and direct links, here the nodes of directional links are identified " $u$ " and nodes with direct links are identified as "v", these nodes are connected by links "L".

The network graph $\mathrm{G}$ for proposed work is identified as $G=(U, V, L)$. As stated in proposed work, every node have interference area up to second hop of directional antennas " $u$ " and " $\mathrm{u}+1$ " and up to single hop for direct connected node " $\mathrm{v}$ " (as the directed node is in different direction of directional antenna). Using neighbouring directed coloring graph the identified interference zone is expressed as $\mathrm{Iz}=(\mathrm{u}, \mathrm{u}+1, \mathrm{v})$. For our work, we modeled 2.4 $\mathrm{GHz}$ backhaul via OPNET 14. The system is designed with two scenarios.

- Allocation of non-orthogonal channels to interference zones of WMN backhaul

- Allocation of orthogonal channels to interference zones of WMN backhaul.

Both scenarios comprise Multiple Access points, every access point have 3 interfaces, 2 interfaces are reserved for backhaul link, there for it comprises 2 interference zones. The third interface is used for user access. Running application for system is FTP, which serves users in uniform pattern. In 1st scenario the channels are randomly assign to all nodes of zones without any care of orthogonal channels. While in 2nd scenario the group of orthogonal channels formatted by proposed mathematical process assigned to different interference zones. This maintains the orthogonality of channels within zones resulting minimum interference.

\section{A. Greedy Algorithm for $2.4 \mathrm{GHz}$ backhaul link}

Following section illustrates the pseudocode of greedy algorithm for channel assignment of $2.4 \mathrm{GHz}$ backhaul links.
The core idea is to select, identified interference links and set of orthogonal channels, then assign channels to every link of in interference. That procedure will repeat until channels assigned to complete network. Every time selected interference links and channel set must be different. With this the network sustains orthogonality to reduce channel interference.

\section{B. Algorithm Greedy channel Assign} for $2.4 \mathrm{GHz}$ backhaul

Input: Graph $\mathrm{G}=(\mathrm{U}, \mathrm{V}, \mathrm{E})$; Available channels 1, 2, 3, • $\bullet, 11$

1) Find conflict links Ec for each node $v$ belongs to $\mathrm{G}=(\mathrm{U}, \mathrm{V}, \mathrm{E})$

2) For each interference zone $\mathrm{Iz}=(\mathrm{u}, \mathrm{u}+1$, $v)$, select set of orthogonal channels of channel " $n$ " by, $\mathrm{On}=\mathrm{UN} \mathrm{m}=1=\{|(\mathrm{m}-\mathrm{n})|$ $>4\}$

3) Assign channel, member of $\mathrm{On}=u j$,

4) Assign channel, member of On $=u j+1$,

5) Assign channel, member of $\mathrm{On}=\mathrm{Vi}$,

6) Iz $6=\mathrm{Iz}$ of (1) and Orthogonal channels for $n 6=n$ of $(1)$

7) Repeat from 1 to 5 for every node belongs to $\mathrm{G}$

8) end

\section{Results and Discussion}

Proposed work analyzed the throughput and delay of orthogonal and non-orthogonal channel schemes for interference zones. Figure 2 presents the comparative results of two scenarios in terms of average throughput, throughput of both scenarios of system is in quiet mode up to $50 \mathrm{sec}$, which is time taken by server to respond to users.

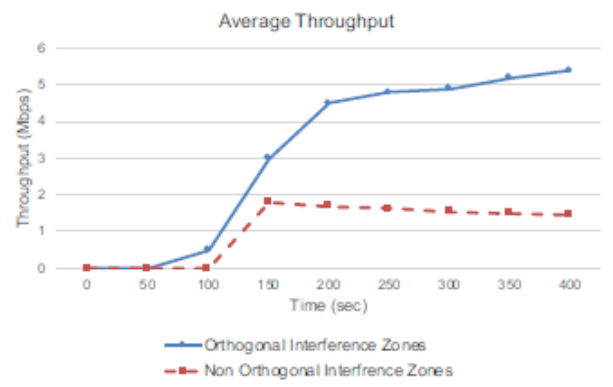

Fig. 2. Average throughput of both schemes 
6 and 7 presents the plots for normal distribution of both scenarios

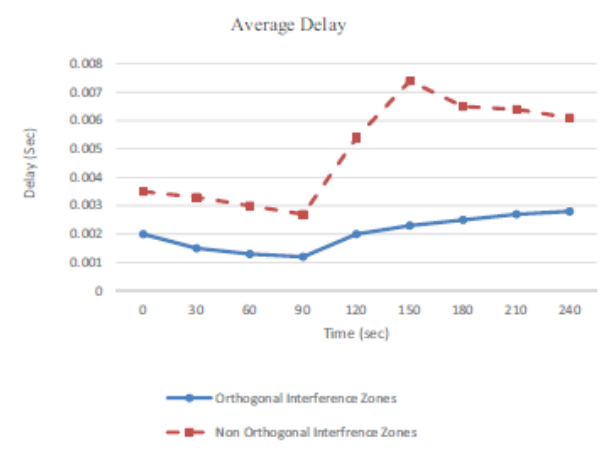

Fig. 3. Average delay of both schemes

Fig. 2 illustrate the association of both scenarios by average throughput, this comparison shows that non-orthogonal allocation throughput drops after certain events while throughput of orthogonal allocation is constantly increasing, further it is observed that performance of proposed scheme sweeps $70 \%$ more than nonorthogonal.

Fig: 3 expressed the performance of both scenarios in terms of average delay, it shows that the maximum delay for

orthogonal interference zones is less than $0.003 \mathrm{sec}$, while for some events of nonorthogonal interference zones, the delay reaches to $0.008 \mathrm{sec}$. Approximately we can reduce the delay in system by half with orthogonal interference zones.

This can be further justified from Fig. 4 and 5 , where the average throughput and average delay is plotted with number of users. The number of users varies with the difference of 10 and provides 10 different points to observe the results. Both results show the considerable enhancement in system performance when we use the proposed channel assignment scheme for backhaul links. With respect to simulation analysis, the simulation model is

repeated for multiple times with different number of users and find out the different confidence intervals. The Table II shows the statistical data for two scenarios of $2.4 \mathrm{GHz}$ backhaul. For the given statistical values, Fig.

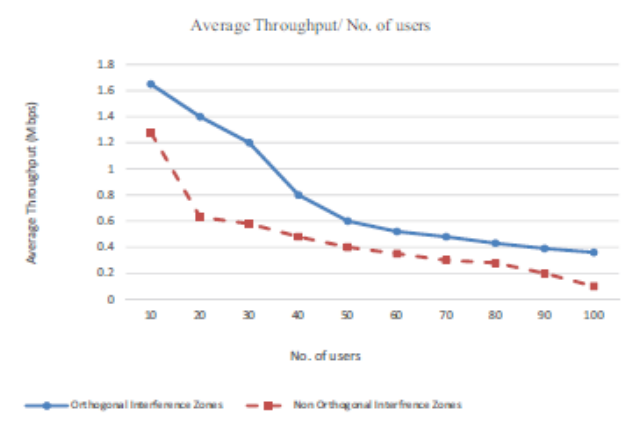

Fig. 4. Average throughput/No. of users of both schemes

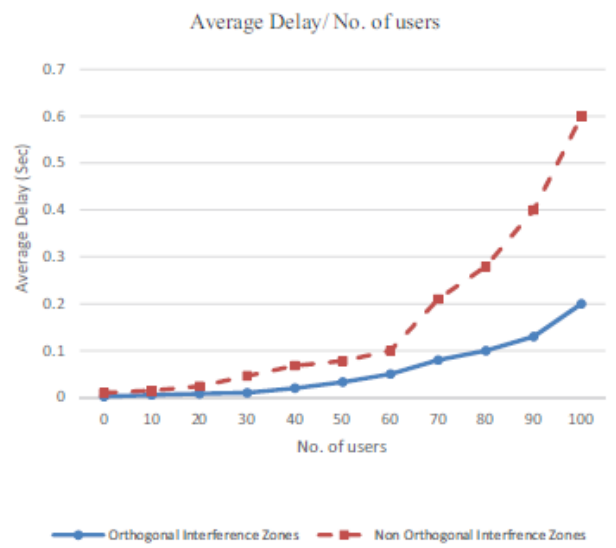

Fig. 5. Average delay / No. of users of both schemes

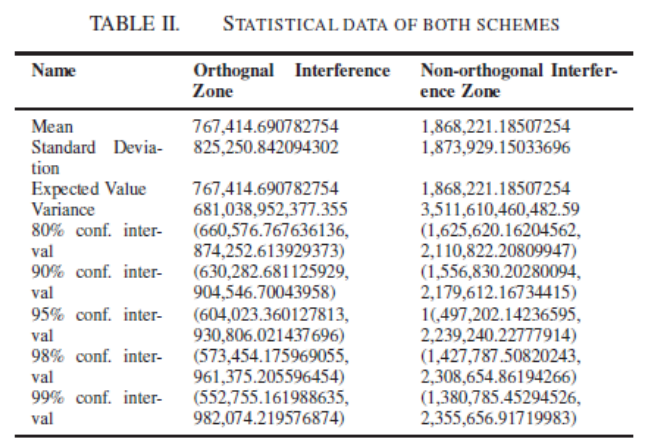




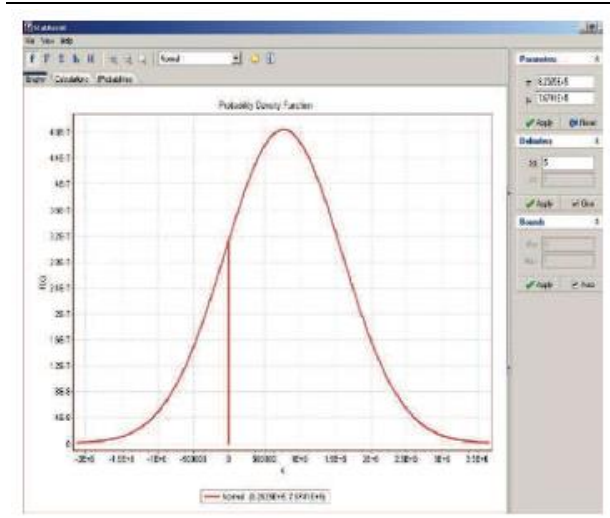

Fig. 6. Normal distribution of orthogonal interference zone

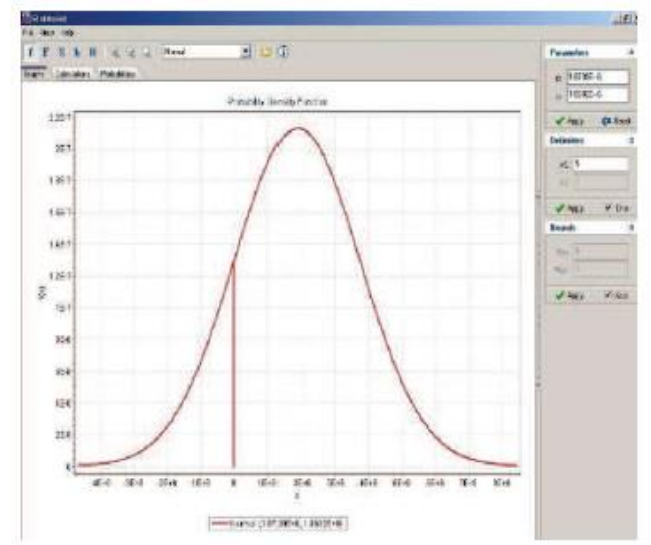

Fig. 7. Normal distribution of non-orthogonal interference zone

\section{Conclusion}

An effective channel assignment scheme is presented where channels of $2.4 \mathrm{GHz}$ are statically arranged as orthogonal channels to the Inter flow and Intra flow links of WLAN backhaul. Co-channel interference from 360 degrees is initially controlled by directional antennas, further the proposed channel arrangement sustaining orthogonality between direct (inter-flow) and directional (Intra-flow) links, this reduces the co-channel interference among these nodes.

In suggested work the interference is controlled at two phases: $1^{\text {st }}$ by reducing interference directions (degrees), $2^{\text {nd }}$ by allocating orthogonal channels to selected links. This study found significant improvement in system performance with the increase in system throughput and reduction in delay. Simulated results clearly illustrate that orthogonal scheme reaches approximately twice the performance in comparison with the non-orthogonal scheme.

\section{REFERENCES}

[1] Mannava, P., 2018. A Comprehensive Study on The Usage of Big Data Analytics for Wireless and Wired Networks. International Journal of Scientific Research in Science and Technology (IJSRST), Online ISSN: 2395602X, Print ISSN: 2395, 6011, pp.724-732.

[2] Pang, Z., Luvisotto, M. and Dzung, D., 2017. Wireless high-performance communications: The challenges and opportunities of a new target. IEEE Industrial Electronics Magazine, 11(3), pp.20-25.

[3] Papavassiliou, S., Tsiropoulou, E.E., Promponas, P. and Vamvakas, P., 2020. A Paradigm Shift Toward Satisfaction, Realism and Efficiency in Wireless Networks Resource Sharing. IEEE Network.

[4] Tabata, H., Suganuma, H. and Maehara, F., 2021. Impact of adjacent-channel interference on transmission performance in asynchronous FBMC/OFDM systems. IEICE Communications Express, p.2020XBL0183.

[5] Iqbal, S., Abdullah, A.H. and Qureshi, K.N., 2020. An adaptive interference-aware and traffic-aware channel assignment strategy for backhaul networks. Concurrency and Computation: Practice and Experience, 32(11), p.e5650.

[6] Harkusha, S. and Yevdokymenko, M., 2021. The Development of Routing Flow Model in IEEE 802.11 Multi-radio Multi-channel Mesh Networks, Shown as a Konig Graph. In DataCentric Business and Applications (pp. 513528). Springer, Cham.

[7] Hou, Y., Li, M. and Zeng, K., 2017, January. Throughput optimization in multi-hop wireless networks with reconfigurable antennas. In 2017 International Conference on Computing, Networking and Communications (ICNC) (pp. 620-626). IEEE.

[8] Jeffrey G. Andrews, Arunabha Ghosh, and Rias Muhamed. Fundamentals of WiMAX: Understanding Broadband Wireless Networking. Prentice Hall, 2007. page 269365.

[9] W. Zhou, X. Chen, and D. Qiao. Practical routing and channel assignment scheme for mesh networks with directional antennas. In IEEE International Conference on Communications, pages 3181-3187, May 2008. 
[10] S. Abbasi, Q. Kalhoro, and M. A. Kalhoro. Efficient use of partially overlapped channels in $2.4 \mathrm{ghz}$ wlan backhaul links. In International Conference on Innovations in Information Technology, pages 7-11, April 2011.

[11] Arunesh Mishra, Vivek Shrivastava, Suman Banerjee, and William Arbaugh. Partially overlapped channels not considered harmful. ACM SIGMETRICS Performance Evaluation Review, 34(1):63-74, June 2006.

[12] Z. Feng and Y. Yang. How much improvement can we get from partially overlapped channels? In 2008 IEEE Wireless Communications and Networking Conference, pages 2957-2962, March 2008.

[13] Liu, K., Li, N. and Liu, Y., 2017, December. Min-interference and connectivity-oriented partially overlapped channel assignment for multi-radio multi-channel wireless mesh networks. In 2017 3rd IEEE International Conference on Computer and Communications (ICCC) (pp. 84-88). IEEE.

[14] Arunesh Mishra, Suman Banerjee, and William Arbaugh. Weightedcoloring based channel assignment for wlans. ACM SIGMOBILEMobile Computing and Communications Review, 9(3):19-31, July 2005.

[15] Shahmirzadi, M.A., Dehghan, M. and Ghasemi, A., 2018. An optimization framework for multicasting in MCMR wireless mesh network with partially overlapping channels. Wireless Networks, 24(4), pp.1099-1117.

[16] 11 b Al-rimy, B.A.S., Kamat, M., Ghaleb, F.A., Rohani, M.F., Abd Razak, S. and Shah, M.A., 2020. A user mobility-aware fair channel assignment scheme for wireless mesh network. In Computational Science and Technology (pp. 531-541). Springer, Singapore.

[17] Derdouri, L., Pham, C., Zouaoui, E.M.E.A. and Zeghib, N., 2019. Performance analysis of self-organised multicast group in multi-radio multi-channel wireless mesh networks. IET Communications, 14(4), pp.693-702.
[18] V. Ramamurthi, A. Reaz, S. Dixit, and B. Mukherjee. Directionality as needed achieving connectivity in wireless mesh networks. In 2008 IEEE International Conference on Communications, pages 30553059, May 2008.

[19] W. Zhou, X. Chen, and D. Qiao. Practical routing and channel assignment scheme for mesh networks with directional antennas. In 2008 IEEE International Conference on Communications, pages 3181-3187, May 2008.

[20] K. Zhou, X. Jia, L. Xie, Y. Chang, and X. Tang. Channel assignment for wlan by considering overlapping channels in sinr interference model. In International Conference on Computing, Networking and Communications (ICNC), pages 1005-1009, Jan 2012.

[21] Gimenez-Guzman, J.M., Crespo-Sen, D. and Marsa-Maestre, I., 2020, December. A Cluster-Based Channel Assignment Technique in IEEE 802.11 Networks. In Telecom (Vol. 1, No. 3, pp. 228-241). Multidisciplinary Digital Publishing Institute.

[22] W. Wang, B. Liu, M. Yang, J. Luo, and X. Shen. Max-cut based overlapping channel assignment for 802.11 multi-radio wireless mesh networks. In Proceedings of the 2013 IEEE 17th International Conference on Computer Supported Cooperative Work in Design (CSCWD), pages 662-667, June 2013.

[23] G. S. Uyanik, M. J. Abdel Rahman, and M. Krunz. Optimal guardband-aware channel assignment with bonding and aggregation in multichannel systems. In 2013 IEEE Global Communications Conference (GLOBECOM), pages 4769-4774, Dec 2013.

[24] Jun Xu, Chengcheng Guo, and Jianfeng Yang. Interference-aware. greedy channel assignment in multi-radio multi-channel wmn. In 25th Wireless and Optical Communication Conference (WOCC), pages 1-4, May 2016 